\title{
Combination of an improved FRF-based substructure synthesis and power flow method with application to vehicle axle noise analysis
}

\author{
C.Q. Liu \\ DaimlerChrysler Corporation, CIMS 481-47-10, 800 Chrysler Drive, Auburn Hills, MI 48326-2757, USA \\ E-mail: c131@daimlerchrysler.com
}

Received 17 January 2006

\begin{abstract}
In this paper, an improved FRF-based substructure synthesis method combined with power flow analysis is presented and is used for performing a vehicle axle noise analysis. The major transfer paths of axle noise transmitted from chassis to vehicle body are identified and ranked based on power flows transmitted through bushings between the chassis and body. To calculate the power flows, it is necessary to know the reaction forces and the vibrations at the bushing locations on the body side. To this end, the body is represented in terms of experimentally derived frequency response functions (FRF's) at the bushing locations, and the FRF's are coupled with the FEA model of the chassis for performing a total system dynamic analysis. This paper also describes how the FRF's of the vehicle body and the frequency dependent stiffness data of the bushings can be combined together with a simple formulation to better represent the dynamic characteristics of a full vehicle. A classical example is used to illustrates the concept of the method, and the method is then applied to a vehicle axle noise analysis with detailed procedure. The theoretical predictions are compared with experimentally measured results. Good correlation has been obtained.
\end{abstract}

\section{Introduction}

Simplistically, the "power flow" is a "product of force and velocity" or a "work rate" which in tern is an energy rate. It is increasingly accepted that the total power flow is the single parameter that best represents the effectiveness of a vibration isolation system as compared with the traditional measure in terms of the force or displacement transmissibility [3-5,7,10-12]. To calculate the power flow, it is necessary to know the reaction forces and vibration velocities at the isolator locations on the receiver side. It turns out to be a rather arduous task for a vehicle system. The main reason for this lies in the fact that the automotive chassis and body are a large complex system, the reaction forces and vibration velocities depend not only on the strength of excitation within the chassis but also on the coupling of the chassis and the auto body. Moreover, the FEA model of the auto body can not be modeled very well up to mid-frequency range due to the higher modal density. In this paper, the reaction forces and vibration velocities of the body at the locations of bushing are calculated by using an improved hybrid FRF-based substructure synthesis method. That is, the auto body is represented in terms of experimentally derived frequency response functions (FRF's) at the bushing locations, and the FRF's are coupled with a FEA model of chassis for performing a total system dynamic analysis with direct modal frequency response analysis method. This method was first introduced by Klosterman [1,2], since then many authors have attempted to represent various substructures in a total system dynamic analysis $[6,8,9]$. This technique is attractive since dynamic behavior of the total system can be predicted by analyzing and combining the dynamic behavior of simpler substructures. The FRF's of the auto body can be obtained by either experimental test or finite element method. Once the FRF's of the body have been made available, the effects of changes in the chassis on the operating behavior, vibration and noise can be ascertained. Another advantage of this approach is that the order of the final system of equations to be solved is substantially smaller than 
the total number of physical degrees of freedom. Therefore a considerable amount of time and effort of the engineers and designers can be saved.

However, this approach as originally presented requires the elimination of reaction (or "constraint", or "coupling") forces and redundant unknown variables by using the constraint equations which represent the connection conditions of the substructures. This may be tedious, in general, and this is especially the case for the auto body and the chassis. This difficulty was overcome by Liu et al. [8]. Next, to perform power flow analysis to identify the major paths of vibration and noise, one needs to know the reaction forces acting on the auto body by the chassis through the bushings. By using the traditional FRF-based method, after obtaining the responses of the system, one has to use the constraint equations again by back substitution to determine these forces. This might be cumbersome and inefficient for complex systems.

This paper uses an improved FRF-based substructure synthesis method which will circumvent these difficulties. Compared to traditional substructure method, the method presented in this paper has the following advantages: First, the method is more suitable for developing a computer program for application to a generic class of systems; Secondly, the analyst is spared the responsibility for eliminating the reaction forces; Finally, by using this method the reaction forces and the responses of the total system can be calculated efficiently.

The paper is divided into four parts. The first part contains the theory of the method and solution method. The second part provides a classical example to illustrate the validity of the method. The third part provides the procedure of application of the method to a vehicle axle noise analysis. Concluding remarks are given in the final part.

\section{Theory}

The net power flow at a point is given in terms of the instantaneous force $F_{i}$ and velocity $v_{i}$ as

$$
P=\frac{1}{T} \int_{0}^{T} F_{i} v_{i} d t
$$

If the force and velocity are assumed to have a harmonic time dependence, $F_{i}=F e^{i \omega t}, V_{i}=V e^{i \omega t}$, then the time-averaged power flow can be written as $[10,12]$

$$
\begin{aligned}
P & =(1 / 2) \operatorname{Re}\left(F^{*} V\right) \\
& =(1 / 2) \operatorname{Re}\left(F V^{*}\right) \\
& =\left((1 / 2)|F|^{2} \operatorname{Re}(M)\right. \\
& =(1 / 2)|V|^{2} / \operatorname{Re}(M)
\end{aligned}
$$

where $M=V / F$ is the complex mobility at the point, the superscript * denotes the conjugate transpose.

In the following, we will focus on how to calculate the reaction forces and vibration velocities of a system composed by two sub-structures.

Consider a structure as shown in Fig. 1. The sub-structure $A$ and $B$ are connected rigidly. We can think $A$ and $B$ as of chassis and automotive body, respectively. For sub-system $A$, we have the following equations of motion:

$$
\left[\begin{array}{ll}
Z_{r r}^{A} & Z_{r c}^{A} \\
Z_{c r}^{A} & Z_{c c}^{A}
\end{array}\right]\left\{\begin{array}{l}
X_{r}^{A} \\
X_{c}^{A}
\end{array}\right\}=\left\{\begin{array}{l}
F_{r}^{A} \\
F_{c}^{A}
\end{array}\right\}
$$

where the subscript $c$ represents a number of coupling points; the subscript $r$ represents the other response points. $X$ 's are response vector; $F$ 's are force vector; $Z$ 's are impedance matrices. For the coupling points on the sub system $B$, the equation of motion can be written as the form

$$
\left[\begin{array}{ll}
0 & 0 \\
0 & Z_{c c}^{B}
\end{array}\right]\left\{\begin{array}{l}
X_{r}^{A} \\
X_{c}^{B}
\end{array}\right\}=\left\{\begin{array}{l}
0 \\
F_{c}^{B}
\end{array}\right\}
$$

The boundary conditions can be written as 


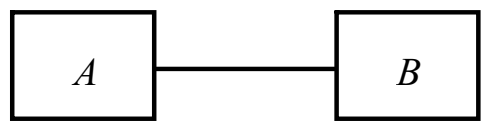

Fig. 1. Subsystems $A$ and $B$ are rigidly connected.

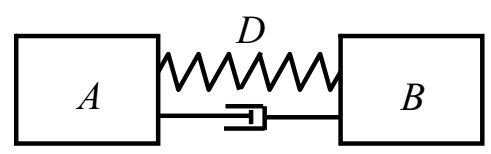

Fig. 2. Subsystems $A$ and $B$ are connected by a number of dampers (springs and dashpots).

$$
\left\{X_{c}^{A}\right\}=\left\{X_{c}^{B}\right\}, \quad\left\{F_{c}^{A}\right\}+\left\{F_{c}^{B}\right\}=\{0\}
$$

Hence, adding Eqs (3) and (4), we obtain

$$
\left[\begin{array}{l}
Z_{r r}^{A} Z_{r c}^{A} \\
Z_{c r}^{A} Z_{c c}^{A}+Z_{c c}^{B}
\end{array}\right]\left\{\begin{array}{l}
X_{r}^{A} \\
X_{c}^{A}
\end{array}\right\}=\left\{\begin{array}{l}
F_{r}^{A} \\
0
\end{array}\right\}
$$

Eq. (6) provides a basis for the total system dynamic analysis with direct modal frequency response analysis. Specifically, the FRF's of the sub system $B$ at the coupling points can be experimentally determined. The inversion of the FRF's is impedance matrix $Z_{c c}^{B}$, which can be calculated by using the Singular Value Decomposition method [9]. Then the response of the total system can be determined by solving Eq. (6).

Observe that, the above method is valid only for the case where the substructures are rigidly connected. To extend this method to a general case, consider a structure shown in Fig. 2, where the sub-systems $A$ and $B$ are connected by several dampers. Here the "damper" means spring and dashpot for simplicity (in the Fig. 2 only one damper is shown). In this case, Eq. (6) is not suitable. The equation for determining the response of the total system can be derived as follows: For sub-system $A$, Eq. (3) is still valid. For the dampers $D$ we have

$$
\left\{X_{c}^{A}\right\}-\left\{X_{c}^{B}\right\}=\left[H^{D}\right]\left\{F_{c}^{B}\right\}
$$

or

$$
\left\{X_{c}^{A}\right\}=\left\{X_{c}^{B}\right\}+\left[H^{D}\right]\left\{F_{c}^{B}\right\}
$$

or

$$
\left\{X_{c}^{A}\right\}=\left[H_{c c}^{B}+H^{D}\right]\left\{F_{c}^{B}\right\}
$$

Hence

$$
\left[H_{c c}^{B}+H^{D}\right]^{-1}\left\{X_{c}^{A}\right\}=\left\{F_{c}^{B}\right\}=-\left\{F_{c}^{A}\right\}
$$

Combining Eqs (3) and (8), we obtain

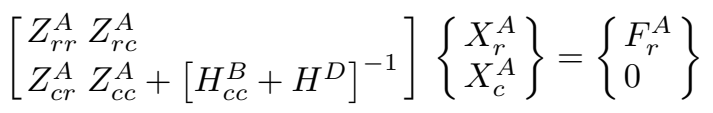

Equation (9) forms a basis for the dynamic analysis of the total system. Specifically, the procedure can be outlined as following:

(1) Determine the stiffness matrix of the dampers (force over displacement, or F/X) experimentally.

(2) Invert the stiffness matrix of the dampers to obtain the frequency response functions, $H^{D}$.

(3) Add the FRF's of the auto body and the dampers together to obtain, $H_{c c}^{B}+H^{D}$.

(4) Invert the combined FRF's matrix, $H_{c c}^{B}+H^{D}$, by using the Singular Value Decomposition method.

(5) To convert the combined stiffness matrices in the format of an external superelement contained within a database file.

(6) Perform the actual coupled analysis based on Eq. (9). 


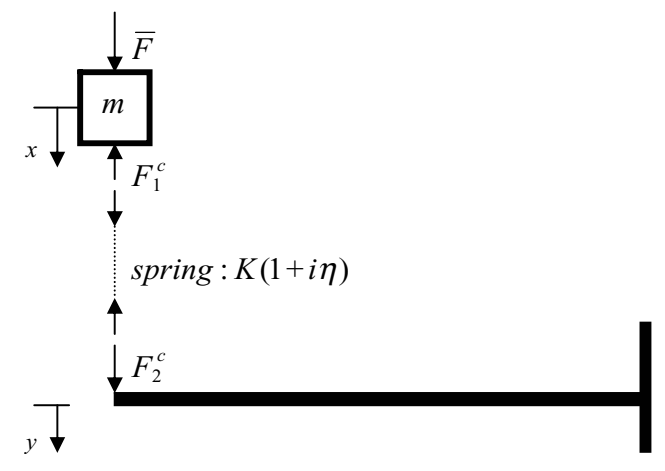

Fig. 3. Model of connected mass, spring and finite beam.

Once the solutions of Eq. (9) are obtained, the reaction (coupling) force vector at the coupling points can be determined from Eq. (7). That is

$$
\left\{F_{c}^{B}\right\}=\left[H_{c c}^{B}+H^{D}\right]^{-1}\left\{X_{c}^{A}\right\}
$$

This reaction force vector can be used to calculate the responses of the subsystem $B$ with the following equation:

$$
\left\{X_{r}^{B}\right\}=\left[H_{c r}^{B}\right]\left\{F_{c}^{B}\right\}
$$

where $H_{c r}^{B}$ is the transfer function matrices between the auxiliary response points and the coupling points within the sub-system $B$. Then, the power flows can be calculated as

$$
P=(1 / 2) \operatorname{Re}\left[\left\{F_{c}^{B}\right\}^{*}\left\{i \omega X_{r}^{B}\right\}\right]
$$

\section{An illustrative example}

In this section, a classical example is given to show the validity of the proposed method. This example was given by Pinnington and White [12] as shown in Fig. 3, in which the mass $m$ was attached with a spring to the free end of the beam; while another end of the beam was attached to an arbitrary structure. The objective is to calculate the power flow transmitted to such a beam from the mass and spring.

The mobility at the attachment point on the beam is given in [12] and may be represented as

$$
\bar{M}=\frac{\bar{V}}{F}=Q_{r}-i Q_{i}-i Q
$$

The expressions of $Q_{r}, Q_{i}$ and $Q$ can be found from Reference [12].

To calculate the power flow, we need to calculate the reaction force at the attachment point on the beam. This can be calculated by using Eq. (10), which in this example becomes

$$
F_{2}^{c}=\left[\frac{\bar{M}}{i \omega}+H^{D}\right]^{-1} X
$$

where $H^{D}$ is the dynamic compliance of the spring. That is

$$
H^{D}=\frac{1}{K(1+i \eta)}
$$

The response (displacement) of mass $m$ can be calculated by using Eq. (9), which in this example becomes

$$
\left[-m \omega^{2}+\left(\frac{\bar{M}}{i \omega}+H^{D}\right)^{-1}\right] X=\bar{F}
$$

from which we obtain 


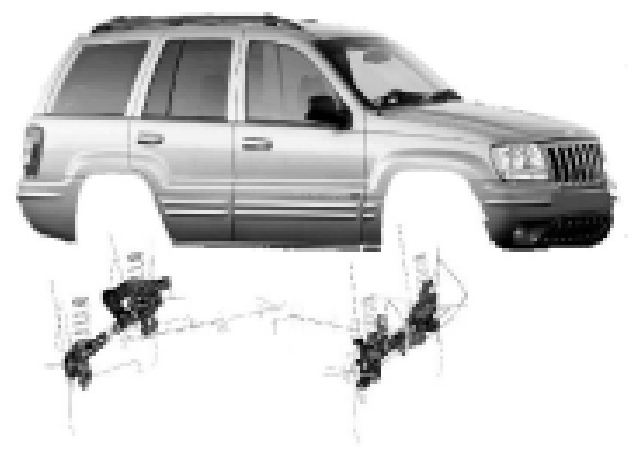

Fig. 4. Vehicle body (above) and FEA model of chassis (below).

$$
X=\left[-m \omega^{2}+\left(\frac{\bar{M}}{i \omega}+H^{D}\right)^{-1}\right]^{-1} \bar{F}
$$

Substituting Eq. (17) into Eq. (14), the reaction force at the attachment point on the beam can be written as

$$
F_{2}^{c}=\frac{\bar{F}}{-m \omega^{2}\left[\frac{\bar{M}}{i \omega}+H^{D}\right]+1}
$$

Substituting Eqs (13) and (15) into Eq. (18), finally we obtain

$$
F_{2}^{c}=\frac{\bar{F}}{i \omega m\left[\omega(i+\eta) / K+i /(\omega m)-i Q-i Q_{i}+Q_{r}\right]}
$$

The power flow can be calculated as

$$
P_{t r}=\frac{1}{2}\left(F_{2}^{c}\right)^{2} \operatorname{Re}(\bar{M})=\frac{1}{2}\left(F_{2}^{c}\right)^{2} Q_{r}
$$

This result is seen to be the same as that of Pinnington and White [12].

\section{Application: Axle noise analysis of a vehicle}

The procedure presented in this paper has been successfully applied to a vehicle axle noise analysis. In this analysis, the body is represented by FRF's at the attachment points with the chassis while the chassis is represented by FEA model as shown in Fig. 4. The main reason for this lies in the fact that because of the relatively high stiffness of the automotive chassis, its FEA model can be modeled very well with finite elements up to the mid-frequency range. This is, however, not true for the body due to higher modal density. Therefore, it is particularly beneficial to use experimental FRF data to represent the body in the mid-frequency range. Next, our main items of interest are the effects of changes in the chassis on the operating behavior. In this case, the FRF representation of the body and the bushings merely acts as a boundary condition for the chassis. Therefore, the degrees of freedom of the final mathematical model are significantly reduced with respect to the total physical degrees of freedom

The total $225 \mathrm{FRFs}(\mathrm{a} / \mathrm{F}$ and $\mathrm{P} / \mathrm{F})$ of the body at the 25 connecting points with chassis were experimentally measured ( $75 \mathrm{a} / \mathrm{F}$ and $150 \mathrm{p} / \mathrm{F})$. Figure 5 shows the test setup. Some typical measured FRF's are shown in Figs 6 and 7. Next, the stiffness of bushings were experimentally measured by using a servo-hydraulic rate test machine rather than detailed FEA models, because it is time consuming to build detailed bushing models. Figure 8 shows two examples of stiffness of the bushings.

The dynamic analysis of the total system was performed according to Eq. (9). In the simulation with NASTRAN SOL 108 , a large mass excitation to enforce $1.17 \times 10^{-3} \mathrm{~mm}$ displacement to simulate the force input from the front pinion to differential gear connection. The input force was calculated as 


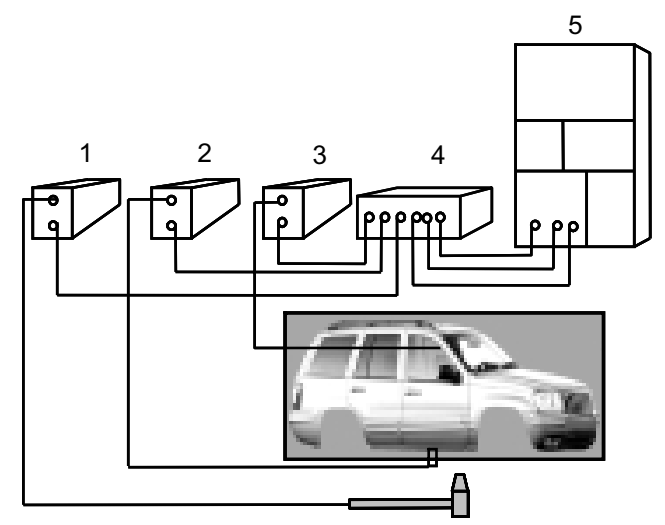

Fig. 5. Test setup for body FRF measurement. 1 - signal generator; 2 and 3 - signal conditioning; 4 - antialiasing; 5 - FFT.

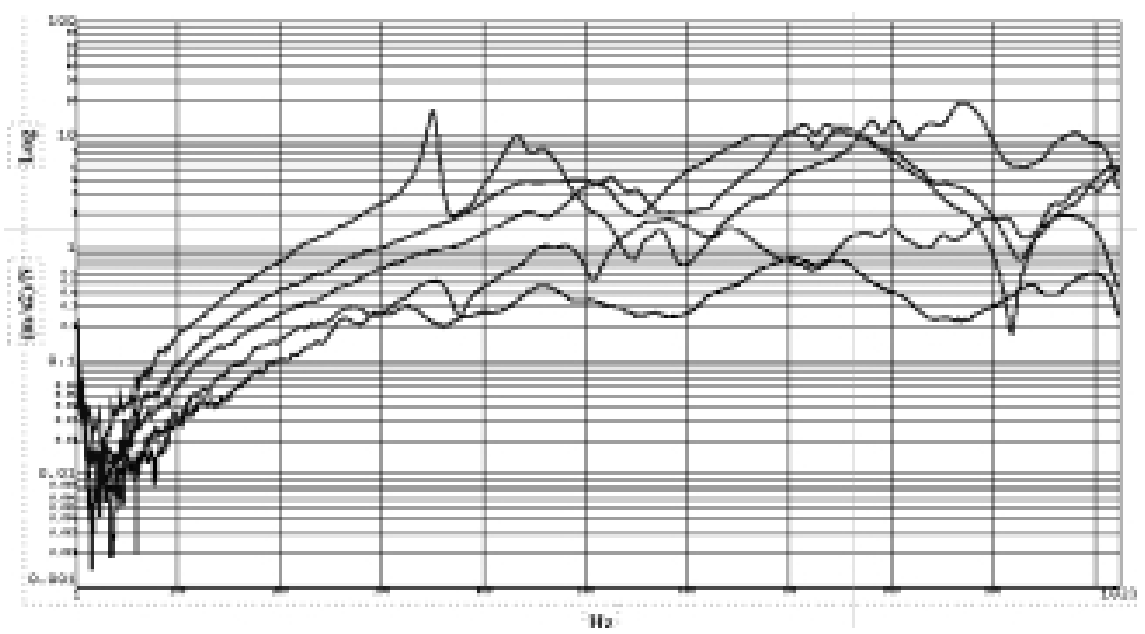

Fig. 6. Examples of FRF's of the vehicle body at the attachment points of bushings.

$$
F=M a=M(2 \pi f)^{2} X
$$

where $M$ is the large mass; $f$ is the frequency; $X$ is the displacement used.

The power flows transmitted from each bushing to auto body were calculated as follows: First, the reaction forces and responses of the body at attachment points of bushing were calculated by using Eqs (10) and (11); then the power flows were calculated by using Eq. (12).

To identify the dominate paths to the axle noise, a 3-D display of power flows of 75 paths and three different frequency bands is shown in Fig. 9, in which the paths (connecting points) identification numbers are denoted by body:1000 to body:1024, respectively, from front to rear of the vehicle. Each of connecting points has three directions ( $\mathrm{x}, \mathrm{y}$ and $\mathrm{z}$ ). The frequency range of interest is divided into three bands: the first band is from 395 to $438 \mathrm{~Hz}$, the second from 438 to $495 \mathrm{~Hz}$, and the third from 530 to $560 \mathrm{~Hz}$. For each frequency band, the power flows are represented by the RMS values (The unit is watt). As it can be seen, the major paths are in the front of the vehicle. Figure 10 is a 2-D display of the power flows for the front 33 paths in $395-438 \mathrm{~Hz}$ frequency band. It can be seen from Fig. 11 that the first three dominate paths in this frequency band are the body:1000:z, body:1005: $\mathrm{z}$ and body:1007:z, which represent, respectively, the vertical direction of the front left shock, front left spring, and front left sway bar attachment points. These results are agree with those from experimental transfer path analysis (TPA) in [8], and also, agree with Operating Deflection Shapes (ODS) animation.

The connecting point ID number from front to rear of the vehicle are body:1000 to body:1024. Each point has x, $\mathrm{y}$, and $\mathrm{z}$ three directions. 


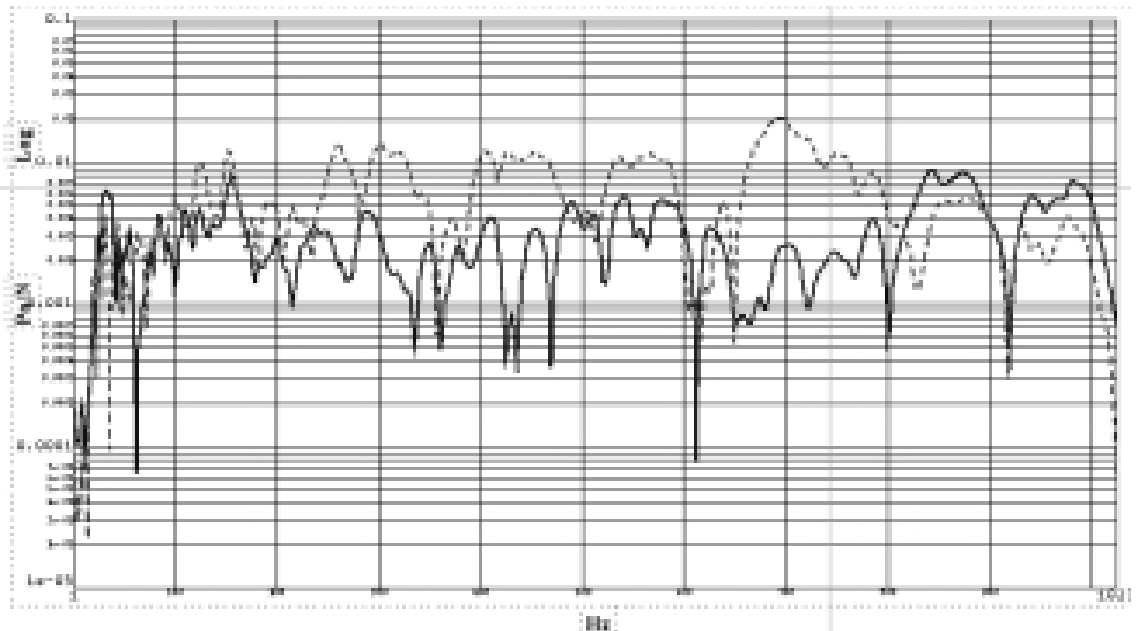

Fig. 7. Examples of acoustic transfer functions $(\mathrm{p} / \mathrm{F})$ of the vehicle body.

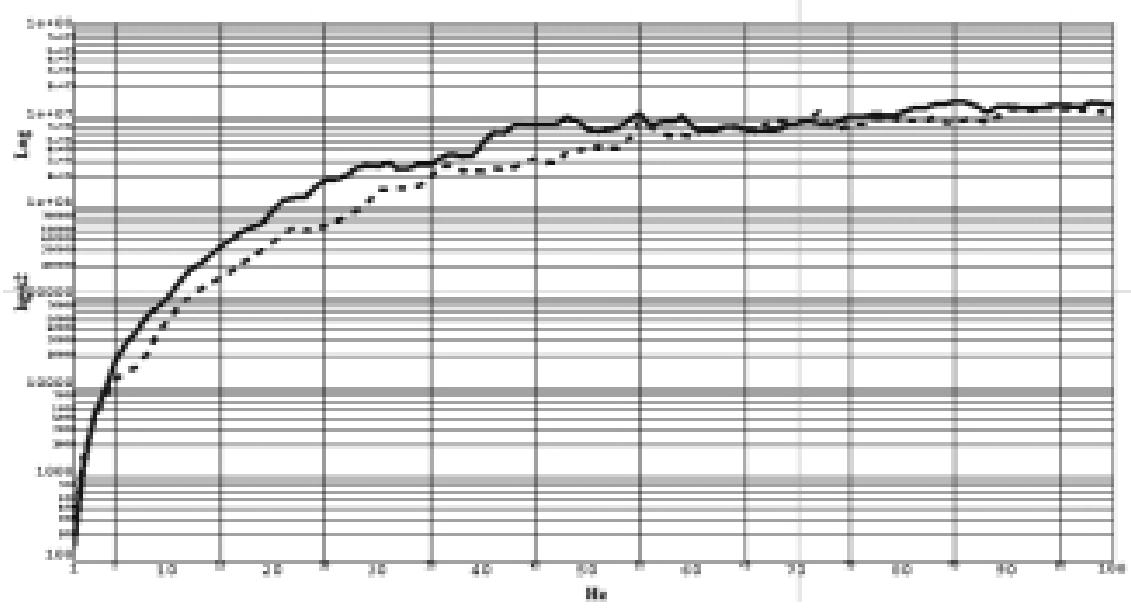

Fig. 8. Examples of frequency dependent stiffness of bushings.

The first three dominate paths are: body:1000:z, body:1005:z and body:1007:z.

Next, as a check, based on the calculated reaction forces at the attachment points, the partial pressure contributions of each path to the sound pressure level at the driver's left ear were calculated. The total sound pressure was calculated as the complex summation of partial contributions of 75 paths by the following formula:

$$
P=\sum_{i=1}^{n}\left(\frac{p}{F}\right)_{i} F_{i}
$$

where $P$ is the total sound pressure at the driver's left ear; $(p / F)_{i}$ is acoustical transfer functions (see Fig. 7), pressure/force; $F_{i}$ is the reaction force calculated at path $i(i=1, \cdots, n=75)$ using Eq. (10). The calculated result and experimentally measured results are shown in Fig. 11, in which the solid line represents the calculated result, and the dot lines are experimentally measured results. To see the variability of test, five different measurements are plotted. As we can see from Fig. 11 that good correlation between the simulation and test results has been obtained. 


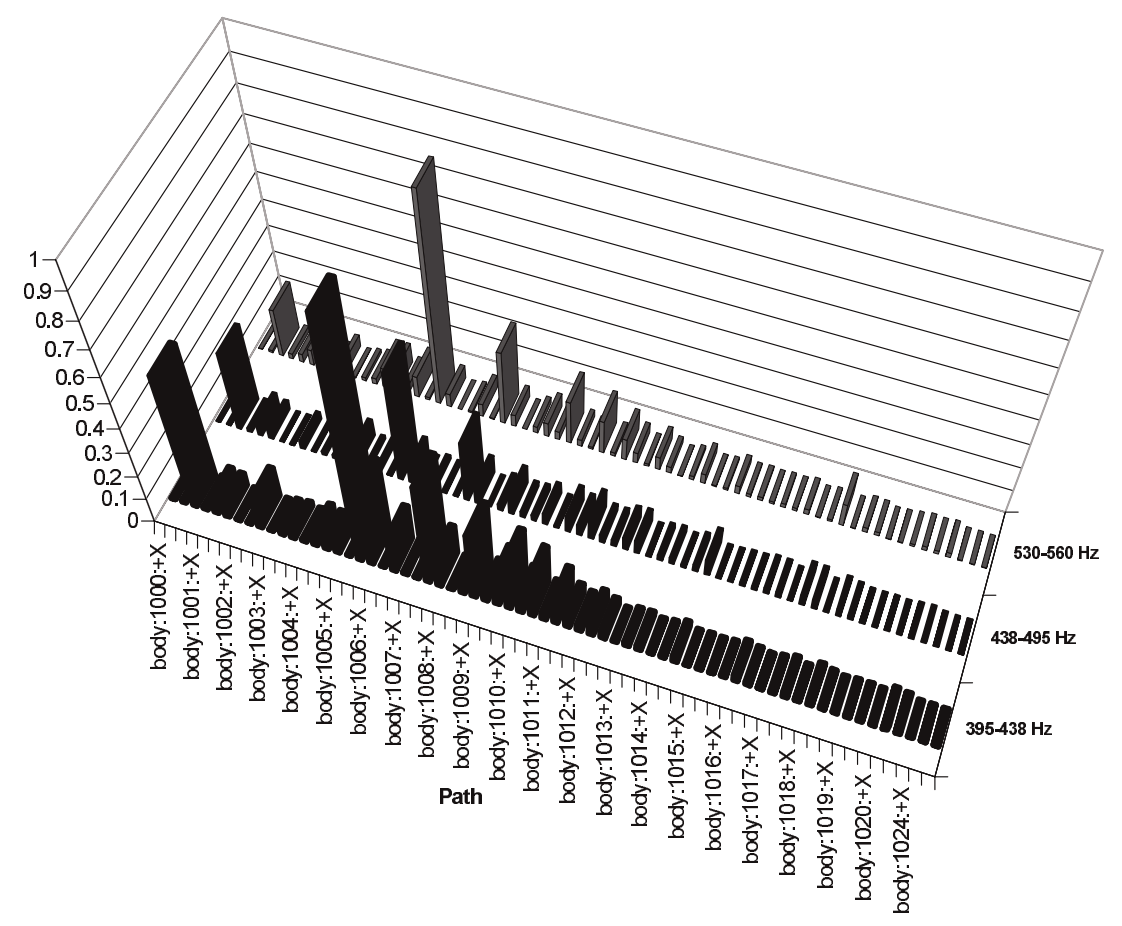

Fig. 9. RMS values of power flows from 75 paths in three frequency bands (Unit: Watt).

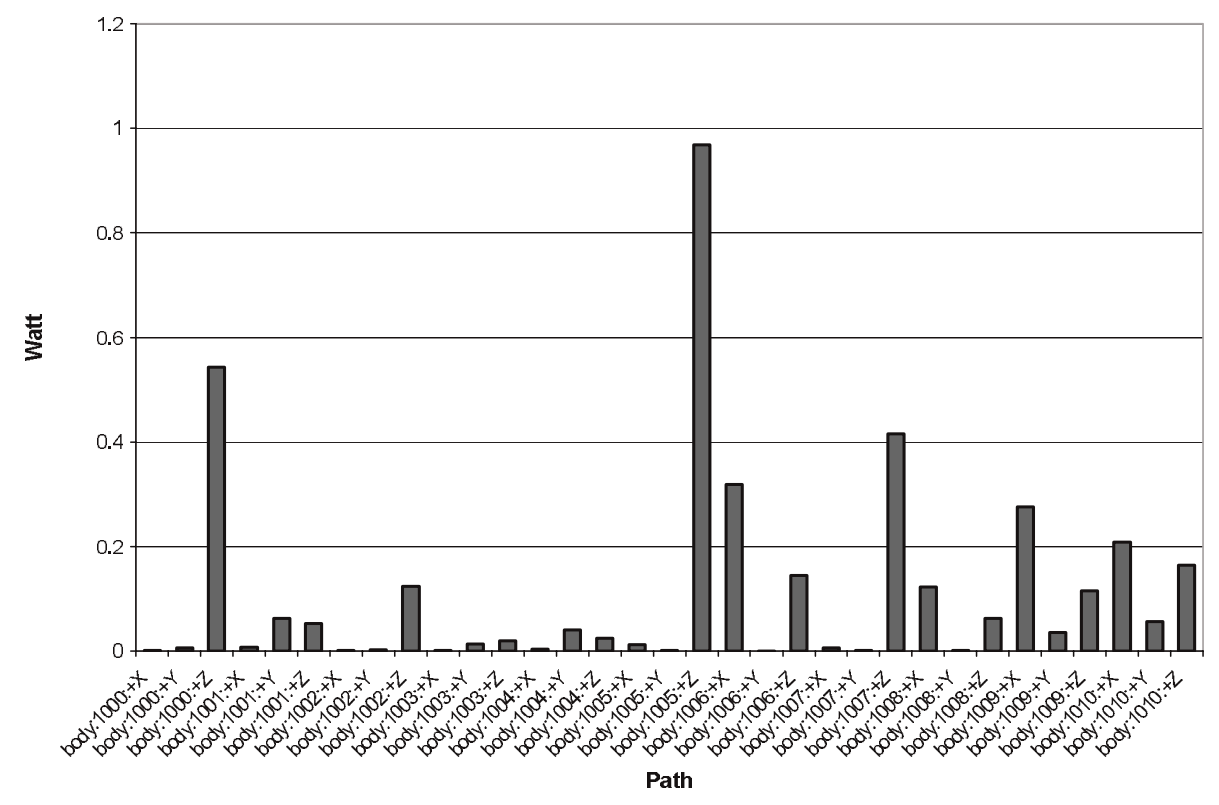

Fig. 10. RMS values of front 33 paths in 395-438 Hz frequency band (Unit: Watt).

\section{Concluding remarks}

An improved FRF-based substructure synthesis method combined with power flow analysis is presented. This method has been successfully applied to a vehicle axle noise analysis. The calculated results are well correlated with test results. 


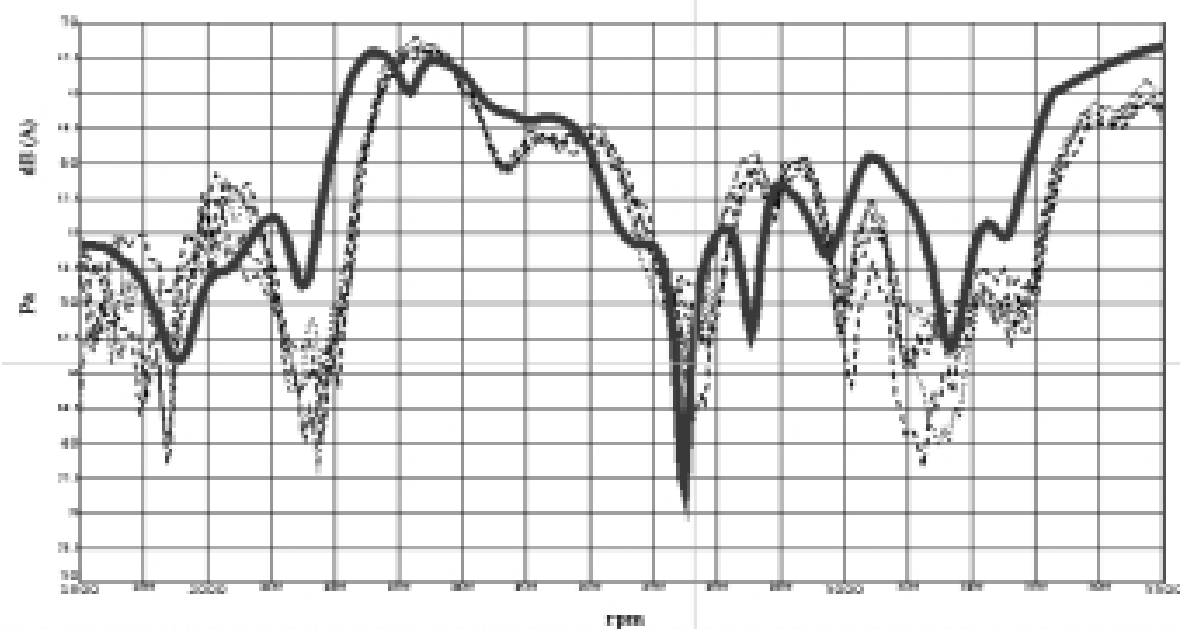

Fig. 11. Sound pressure level at driver's left ear. Calculated result (solid line) vs. 5 experimentally measured results (dot lines).

The improved FRF-based substructure synthesis method is extremely attractive when the main items of interest are the chassis. Once the FRF's of the body have been made available, the effects of changes in the chassis on the operating behavior, vibration and noise can be ascertained efficiently, because the degrees of freedom of the final mathematical model are significantly reduced with respect to the total physical degrees of freedom. In addition, because of the relatively high stiffness of the automotive chassis, its FEA model can be modeled very well with finite elements up to the mid-frequency range. This is however not true for the body due to the higher modal density. Therefore, it is particularly beneficial to use experimental FRF data to represent the body in the middle frequency range.

In comparison with the reaction forces, the power flows are increasingly accepted as more meaningful measure of the effectiveness of a vibration isolation design, especially in mid/high-frequency range. We believe that the method presented in this paper can be used not only to identify the major transfer paths of power flows via isolators efficiently, but also to identify more options and directions regarding how to improve the design.

\section{References}

[1] A.L. Klosterman and J.R. Lemon, Dynamic Design Analysis via the Building Block Approach, Sound and Vibration Bulletin 42, $97-104$. Type any references over these paragraphs.

[2] A.L. Klosterman, On the Experimental Determination and Use of Modal Representations of Dynamic Characteristics, Ph.D. Dissertation, University of Cincinnati, 1971.

[3] Y.K. Koh and R.G. White, Analysis and control of vibrational power transmission to machinery supporting structures subjected to a multi-excitation system, part I: driving point mobility matrix of beams and rectangular plates, Journal of Sound and Vibration 196 (1996), 469-493.

[4] Y.K. Koh and R.G. White, Analysis and control of vibrational power transmission to machinery supporting structures subjected to a multi-excitation system, part II: vibration power analysis and control schemes, Journal of Sound and Vibration 196 (1996), $495-508$.

[5] Y.K. Koh and R.G. White, Analysis and control of vibrational power transmission to machinery supporting structures subjected to a multi-excitation system, part III: vibration power cancellation and control experiments, Journal of Sound and Vibration 196 (1996), 509-522.

[6] M. Kop and M. Brughmans, Tutorial of LMS International for Experimental FRF based MSC.NASTRAN superelements (Gateway 3.5C and MSC.NASTRAN 70.0), LMS International, 09/2000.

[7] W.L. Li, M. Daniels and W. Zhou, Vibrational power transmission from a machine to its supporting cylindrical shell, 257 (2002), $283-299$.

[8] C.Q. Liu and H. Mir, A Hybrid method for Vehicle Axle Noise Simulation with Experimental Validation, 2003 SAE Noise and Vibration Conference and Exhibition, Paper Number: 2003-01-1707.

[9] T. Martens and K. Wyckaet, Matrix inversion technology for vibro-acoustic modeling applications: practical exaples of measurement noise reduction by SVD, LMS International, Leuven, Belgium.

[10] A.T. Moorhouse and B.M. Gibbs, Prediction of the structure-borne noise emission of machines: Development of methodology, Journal of Sound and Vibration 167(2) (1993), 223-237. 
[11] J. Pan, J.Q. Pan and C.H. Hansen, Total power flow from a vibrating rigid body to a thin panel through multiple elastic mounts, Journal of the Acoustical Society of America 92 (1992), 895-907.

[12] R.J. Pinnington and R.G. White, Power flow through machine isolators to resonant and non-resonant beams, Journal of Sound and Vibration 75(2) (1981), 179-197. 

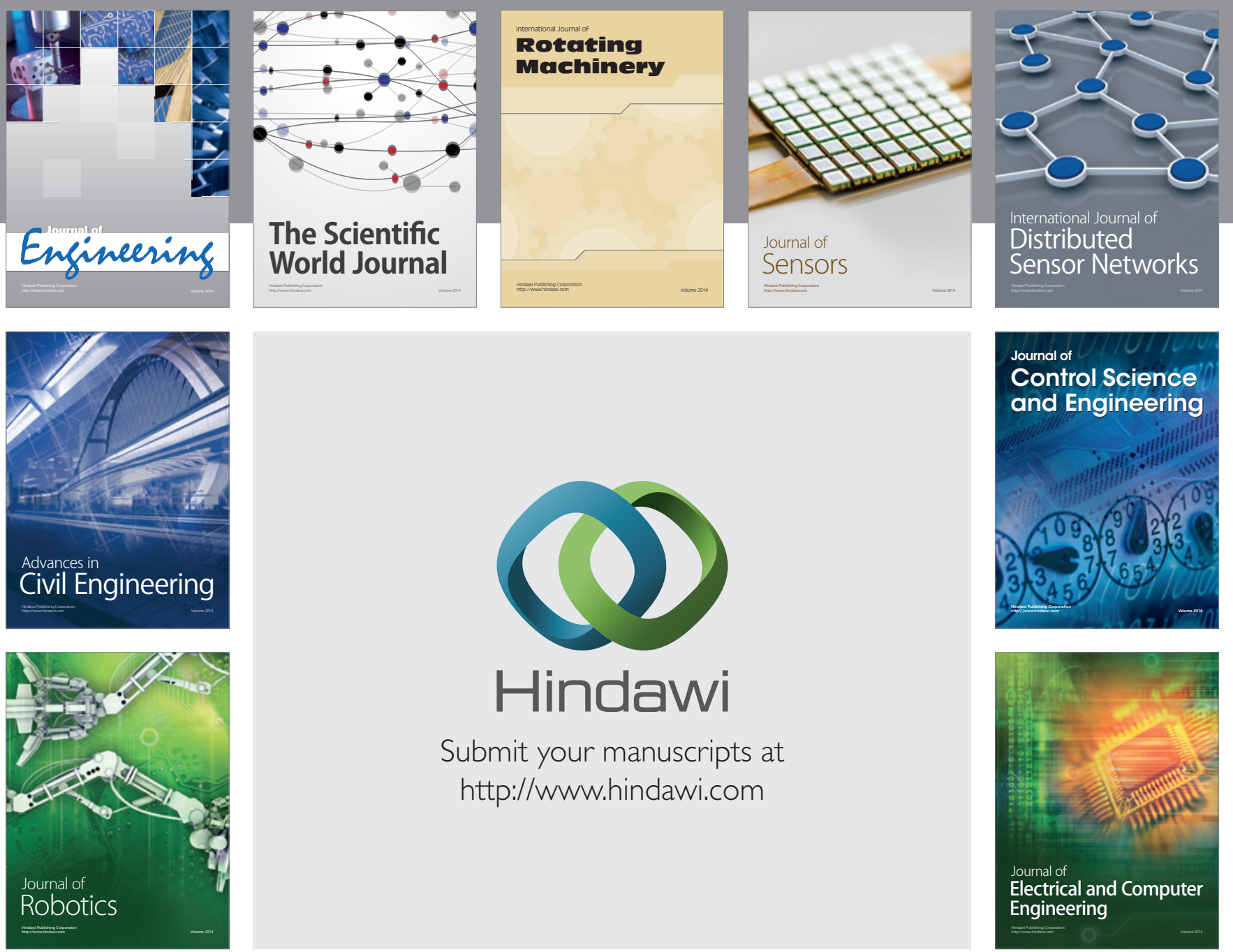

Submit your manuscripts at

http://www.hindawi.com
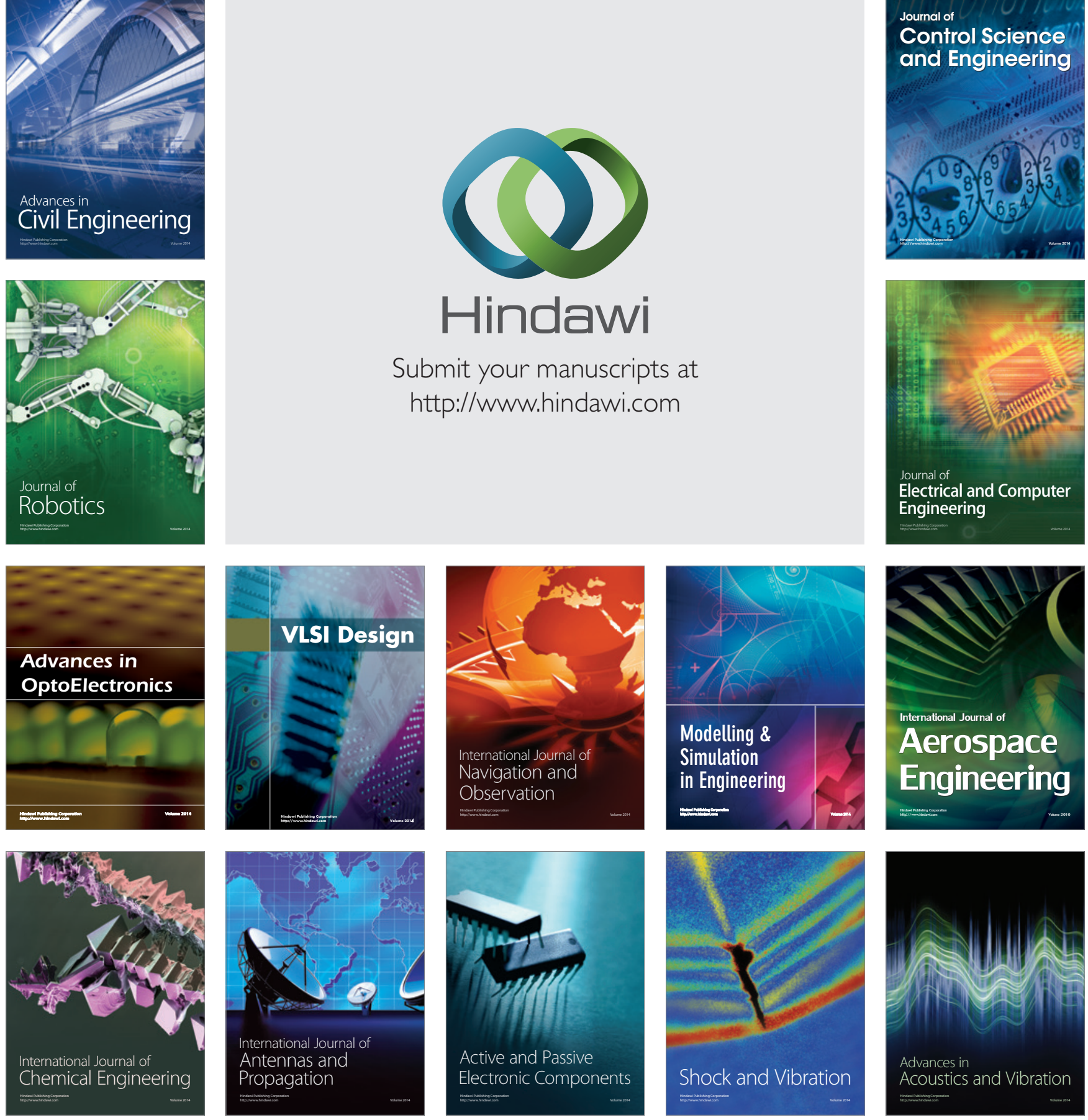